\title{
FERTILITÉ, PROLIFICITÉ ET FÉCONDITÉ PENDANT LA SAISON SEXUELLE DES BREBIS INSÉMINÉES ARTIFICIELLEMENT APRÈS TRAITEMENT A L'ACÉTATE DE FLUOROGESTONE
}

\author{
G. COLAS, J. THIMONIER, M. COUROT et R. ORTAVANT \\ avec la collaboration technique de C. CORnU et Y. GUÉrin \\ Station de Physiologie de la reproduction. \\ Centre de Recherches de Tours, I. N.R.A., \\ Nouzilly B.P.1, 37380 Monnaie
}

\section{RÉSUMÉ}

Nous analysons les conditions les plus favorables à l'obtention d'une bonne fertilité, prolificité et fécondité, des brebis après induction des chaleurs par voie hormonale (acétate de fluorogestone) et insémination artificielle pendant la saison sexuelle.

I 436 femelles de race Ile de France, Préalpes et Lacaune reçoivent une éponge imprégnée de 30 ou $40 \mathrm{mg}$ d'acétate de fluorogestone. Le retrait de l'éponge, effectué le matin, est suivi, d'une injection de o, 40o ou 500 UI de PMSG. Les animaux sont inséminés indépendamment de leur venue en chaleur.

I. Les taux de fertilité et de fécondité à la chaleur induite, sont plus élevés chez les brebis traitées à la PMSG que chez celles recevant le progestogène seul. Les valeurs obtenues sont alors identiques au cours des deux premiers estrus qui suivent le retrait de l'éponge $699,0 \mathrm{p}$. Ioo vs, $7 \mathrm{I}, 8$ p. Ioo et 96,2 vs 95,0$)$. La prolificité augmente, mais d'une manière non significative (139,5 P. 100 vS I32,3 P. I00: $\mathrm{P}>0,05$ ).

2. Les trois critères dépendent également de la dose de FGA administrée : Les valeurs les plus élevées sont obtenues avec $40 \mathrm{mg}$ de FGA

$$
61,7 \text { p. I00 - I6I,0-99,0 p. Io0 }
$$

3. Le moment d'insémination conditionne à la fois la fertilité, la prolificité, et la fécondité. Les meilleurs résultats sont obtenus, lorsque les animaux sont inséminés 48 et 60 heures après le retrait de l'éponge $(69,6 \mathrm{p}$. roo - I 70,8 p. I00 - I I8,0 p. I00).

4. La fertilité augmente, lorsque le volume et le diamètre des paillettes diminuent:

$$
\begin{aligned}
& 0,4 \mathrm{ml}: 64,7 \text { p. 100 } \\
& 0,2 \mathrm{ml}: 73,5 \text { p. I00 } \mathrm{P}>0,05
\end{aligned}
$$

5. Chez la brebis laitière, ces trois critères de reproduction varient avec l'âge : leur valııur est plus élevée chez les sujets les plus jeunes ( 1,5 à 3,5 ans). 


\section{INTRODUCTION}

Les premiers travaux de RoBINson (I965) sur l'administration des progestatifs de synthèse, et les études qui leur ont succédé (RoBINSON et LAMOND, I966 ; WISHART, I967; THImonier et al., I968 $a$ et $b$, etc.), ont abouti à la mise au point de méthodes permettant de déclencher d'une manière satisfaisante une activité sexuelle chez la Brebis.

Cependant, les hormones utilisées perturbent les conditions de progression (QUinlivan et Robinson, I967, I969) et de survie des spermatozoïdes dans les voies génitales femelles (HAWk et CONLEY, I97I). Il s'ensuit une baisse de fertilité pendant l'œstrus consécutif à l'arrêt đu traitement. Par ailleurs, il a été démontré que cette baisse de fertilité est plus accusée après insémination artificielle qu'après accouplement (LINDsay et al., I967; Willemse, BRAND et MuRling, I968). Ce phénomène est d'autant plus gênant que l'on est amené, pour des raisons pratiques, à faire appel à une mise en place artificielle du sperme.

Pour surmonter l'effet dépressif des progestagènes, nous avons été amenés d'une part à améliorer la synchronisation de 1'œstrus induit par le progestatif (administration de PMSG (CoGNIE et al., I968, I970), étude de différentes doses de FGA) d'autre part à rechercher les meilleures conditions d'utilisation de la semence (nombre et moment d'inséminations, conditionnement du sperme) pendant cet œstrus.

Connaissant par ailleurs l'importance de 1'âge de la femelle sur ses capacités de reproduction, nous avons inclus dans notre étude, cet élément de variation.

Nous avons analysé 1'influence de tous ces facteurs sur la fertilité, la prolificité et la fécondité des brebis.

\section{I. - MATÉRIEL, ETT MÉTHODES}

$$
\text { 1. - Animaux expérimentaux }
$$

\section{Femelles.}

Nos essais (I968 à 197I) sont réalisés sur les races à viande Ile de France et Préalpes du Laboratoire pendant leur saison sexuelle (septembre-décembre) et sur la race laitière Lacaune de la région de Roquefort au début et au milieu de sa période habituelle de reproduction (juin-juillet). Les brebis Lacaune, en fin de lactation à l'époque de nos expériences, sont soumises à une traite biquotidienne. Dans tous les cas, les animaux ont agnelé depuis au moins trois mois.

Mâles.

Les béliers, des mêmes races, sont âgés de 8 mois à 6,5 ans. Un plan d'accouplement est établi avant chaque essai de façon à éviter le mélange de races ou d'éjaculats.

En cas de retour en chaleur, les brebis sont toujours réinséminées avec la semence du bélier utilisé lors de la première intervention.

\section{2. - Traitement hormonal}

L'acétate de fluorogestone (1) (30 ou $40 \mathrm{mg}$ ) est administré par voie vaginale (RobINson, 1965) pendant $I_{4}$ jours. Ce traitement est complété, dans certains cas, par une injection intramus-

(1) Acétate de fluorogestone = FGA (I7 $\alpha$ acétoxy-9 $\alpha$ fluoro-I I $\beta$ hydroxy-pregn-4-en 3-20 dione). 
culaire de PMSG au moment du retrait de l'éponge (Brebis : 4 oo UI; Agnelles : 500 UI). Celui-ci a toujours lieu le matin entre $7 \mathrm{~h} 30$ et $9 \mathrm{~h} 30$.

\section{3. - Préparation du sperme}

La récolte (vagin artificiel) a lieu deux fois par jour. Le sperme est soumis aux contrôles habituels (motilité, volume, concentration) avant d'être dilué $\left(625 \times \mathbf{I O}^{6} \mathrm{spz} / \mathrm{ml}\right.$ ou I $25^{\circ} \times$ IO $^{8}$ $\mathrm{spz} / \mathrm{ml}$ ) dans du lait écrémé reconstitué, conditionné en paillettes (fines ou moyennes) et maintenu à la température de $+15^{\circ} \mathrm{C}$ (Colas et al., I968).

Chaque dose de semence renferme $25^{\circ} \times 10^{6}$ spermatozoïdes.

$$
\text { 4. - Mise en place du sperme et conduite des opérations }
$$

Pendant l'œstrus consécutif au traitement, que nous appelons ostrus induit $\left(o_{1}\right)$, nous inséminons les brebis sans contrôle de chaleurs ( 2 ou 3 IA/ $\left.; 250 \times 10^{6} \mathrm{spz} / \mathrm{IA}\right)$, à des moments prédéterminés par rapport au retrait de l'éponge, variables selon les expériences.

Au cours du second cestrus $\left(o_{2}\right)$ elles sont inséminées :

a) ou bien au fur et à mesure de l'apparition de leur chaleur, une fois par jour $\left(25^{0} \times 10^{6}\right.$ $\mathrm{spz} / \mathrm{IA}$ ) pendant 2 jours (Exp. I) ; il s'agit alors de femelles déjà inséminées en $o_{1}$ mais non gestantes.

b) ou bien sans détection préalable des chaleurs, une fois par jour $\left(25^{\circ} \times 10^{6} \mathrm{spz} / \mathrm{IA}\right) \mathrm{du}$ I $7^{\mathrm{e}}$ au $20^{\mathrm{e}}$ jour après le retrait de l'éponge (Exp. II). Ces brebis, qui ne sont inséminées qu'au second cestrus, nous ont servi à comparer la fertilité des deux premières chaleurs consécutives au traitement hormonal. Pour pouvoir faire cette comparaison à la même époque, nous avons décalé de I 7 jours la mise en place des éponges dans les lots " $\mathrm{O}_{1}$ " et " $\mathrm{O}_{2}$ ". Les animaux appartenant aux deux lots étaient pris dans les mêmes élevages et recevaient la semence des mêmes béliers.

\section{5. - Expression et interprétation des résultats}

Ces essais ont été conduits selon les principes de l'analyse factorielle pour les facteurs étudiés (béliers, élevages, traitements, âge des femelles). (I964):

Nous avons utilisé, pour exprimer nos résultats, les définitions de Desvignes et DaRPoux

$$
\begin{aligned}
& \text { Fertilité : } \frac{\text { Nombre de femelles mettant bas }}{\text { Nombre de femelles inséminées }} \times \text { IоO } \\
& \text { Prolificité }: \overline{\text { Nombre de femelles mettant bas }} \times \text { Ioo }
\end{aligned}
$$

Fécondité : $\frac{\text { Nombre d'agneaux }}{\text { Nombre de femelles inséminées }} \times$ roo $=$ Fertilité $\times$ Prolificité

L'analyse statistique (fertilité et prolificité) est faite selon le test $\chi^{2}$ corrigé (Lison, I958) de PEARSON.

\section{II. — RÉSULTATS}

\section{I. - Fertilité après traitement progestatif et fertilité naturelle : Infuence de la PMSG (tabl. I)}

En l'absence de PMSG (tabl. I), la fertilité est plus faible au premier qu'au second œstrus après le retrait de l'éponge $(\mathrm{P}<0, \mathrm{OI})$. La fécondité est également plus basse mais la prolificité n'est pas modifiée. 


\section{TABLEAU I}

Infuence du traitement progestatif (40 $\mathrm{mg}$ FGA - I 4 jours) et de la PMSG (4oo UI), sur la reproduction des brebis inséminées artificiellement pendant la saison sexuelle

$o_{1}$ : Estrus induit par le traitement progestatif.

$\mathrm{o}_{2}$ : Second œestrus après le traitement progestatif.

En $o_{1}$ et en $o_{2}$ (Exp. II), les animaux sont inséminés sans détection préalable des chaleurs

\begin{tabular}{|c|c|c|c|c|}
\hline \multicolumn{2}{|c|}{$\begin{array}{l}\text { Insćmination } \\
\text { effectuée à l'cstrus }\end{array}$} & \multirow{2}{*}{$\begin{array}{l}\begin{array}{c}\text { Fertilité } \\
(\%)\end{array} \\
54,5(311)^{*} \\
72,0_{(11)}\end{array}$} & \multirow{2}{*}{$\begin{array}{c}\begin{array}{c}\text { Prolificité } \\
(\%)\end{array} \\
147,0_{(177)} \\
150,5(91)\end{array}$} & \multirow{2}{*}{$\begin{array}{c}\text { Fécondité } \\
(\%) \\
\\
83,5(311) \\
123,5_{(111)}\end{array}$} \\
\hline $\begin{array}{l}\text { Expérience I } \\
\quad(I . \text { de Fr. } \\
+\quad \text { Préalpes })\end{array}$ & $\begin{array}{l}\mathrm{O}_{1} \text { (sans PMSG) } \ldots \\
\mathrm{O}_{2} \ldots \ldots \ldots \ldots\end{array}$ & & & \\
\hline $\begin{array}{l}\text { Expérience II } \\
\text { (Lacaune) }\end{array}$ & $\begin{array}{l}\mathrm{O}_{1} \text { (avec PMSG) } \ldots \\
\mathrm{O}_{2} \ldots \ldots \ldots \ldots \ldots\end{array}$ & $\begin{array}{l}69,0(\mathbf{1 0 3}) \\
71,8_{(241)}\end{array}$ & $\begin{array}{l}139,\}_{(71)} \\
132,3_{(173)}\end{array}$ & $\begin{array}{l}96,2_{(103)} \\
95,0_{(241)}\end{array}$ \\
\hline $\begin{array}{l}(): \text { Anim } \\
*: P<0\end{array}$ & aux inséminés ou $\mathrm{m}$ & tant bas. & & \\
\hline
\end{tabular}

Par contre, lorsque les brebis reçoivent de la PMSG, les différences de fertilité et de fécondité entre premier et second œestrus disparaissent. La prolificité de $\mathrm{O}_{1}$ augmente par rapport à celle de $\mathrm{O}_{2}$ mais la différence n'est pas significative.

Il est important de noter que les pourcentages de mises-bas en $\mathrm{O}_{2}$ sont identiques dans les expériences I et II, bien que les races et les techniques d'insémination soient différentes.

\section{2. - Dose de FGA}

L'augmentation de la quantité de FGA contenue dans l'éponge entraîne une amélioration de la fertilité $(6 \mathrm{r}, 7$ p. Ioo vs 53,3 p. I00: $\mathrm{P}<0,05)$, de la prolificité $(\mathrm{I} 62,4$ vs $\mathrm{I} 49,5 \mathrm{p}$. Ioo: $\mathrm{P}=0,05$ ) et de la fécondité (Ioo,o vs 79,7$)$ (tabl. 2 et 3 ).

En réalité, comme l'indiquent les résultats ci-après, le pourcentage de mise-bas ne dépend de la dose de FGA que lorsque les brebis ne subissent que deux interventions au cours de l'œestrus induit :

2 IA : 46,5 p. Ioo vs 60 , I p. Ioo (pour 30 et $40 \mathrm{mg}$ respectivement);

3 IA : 63,6 p. I00 vs 63,8 p. IOO ( - - - ) ).

Ceci signifie qu'au-delà de 2 interventions, et avec des doses de $40 \mathrm{mg}$ de FGA, la fertilité n'augmente plus.

Signalons également que l'effet de la quantité de FGA sur la proportion d'animaux mettant bas apparaît aussi bien, mais d'une manière non significative, au début qu'au milieu de la saison de lutte habituelle de la race :

- début de saison (début juin) : 50,0 vs 58,5 p. I00 : $\mathrm{P}>0,05$;

— milieu de saison (fin juin) : 56,7 vs 64,8 p. Ioo : $\mathrm{P}>0,05$. 
TABLEAU 2

Influence de la dose de FGA sur la reproduction des brebis de race Lacaune inséminées artificiellement ( 2 ou 3 IA/ $/$ en $o_{1}$ ) pendant la saison sexuelle

(Les animaux, provenant de Io élevages, reçoivent 400 UI de PMSG au moment du retrait de l'éponge)

\begin{tabular}{|c|c|c|c|}
\hline $\begin{array}{c}\text { Dose FGA } \\
\text { (en } \mathrm{mg} \text { ) }\end{array}$ & $\begin{array}{c}\text { Fertilité } \\
(\%)\end{array}$ & $\begin{array}{c}\text { Prolificité } \\
(\%)\end{array}$ & $\begin{array}{c}\text { Fécondité } \\
(\%)\end{array}$ \\
\hline 30 & $53,3_{(332)} *$ & $149,5_{(178)^{*+}}$ & $79,7_{(332)}$ \\
\hline 10 & $61,7_{(333)}$ & $161,0_{(205)}$ & $99,0_{(333)}$ \\
\hline \multicolumn{4}{|c|}{$\begin{array}{l}*: P<0,05 ; \quad *+: P=0,05 . \\
(\quad): \text { Animaux inséminés ou mettant bas. }\end{array}$} \\
\hline
\end{tabular}

\section{3. - Moment d'insémination}

Pour des raisons d'intérêt pratique et de présentation, nous avons limité 1'expression des résultats aux animaux inséminés deux fois à l'œstrus induit (tabl. 3).

La fertilité, la prolificité et la fécondité sont toujours plus élevées lorsque la mise en place du sperme a lieu 48 et 60 heures après le retrait de l'éponge (pour la dose de $40 \mathrm{mg}$ les valeurs de ces trois critères sont de 69,6-I70,8-I I 8,0). Les écarts entre les trois moments d'IA ne sont cependant significatifs que pour la prolificité $(O, O I<\mathrm{P}$ $<0,05)$. Lorsque les animaux sont inséminés 36 et 60 ou 36 et $4^{8}$ heures après la fin du traitement, leurs performances sont très voisines.

\section{TABLEAU 3}

Infuence du délai retrait de l'éponge (30 et $40 \mathrm{mg}$ )-

insémination sur la reproduction des brebis laitières Lacaune pendant la saison sexuelle

(Les animaux, provenant de so élevages, reçoivent 400 UI au moment du retrait de l'éponge)

\begin{tabular}{|c|c|c|c|}
\hline $\begin{array}{l}\text { Intervalle } \\
\text { Ret. Eponge-IA } \\
\text { (heures) }\end{array}$ & $\begin{array}{c}\text { Fertilité } \\
(\%)\end{array}$ & $\begin{array}{c}\text { Prolificité (1) } \\
(\%)\end{array}$ & $\begin{array}{c}\text { Fécondité } \\
(\%)\end{array}$ \\
\hline 36 et 60 & $50, \pm(131)$ & $14^{\prime}{ }^{\prime}, 0_{(66)}$ & $72,5_{(131)}$ \\
\hline 36 et 48 & $48,8_{(133)}$ & $1 / 49_{(65)}$ & $73,0_{(133)}$ \\
\hline 48 et 60 & $60,5_{(139)}$ & $\left.164^{\prime}, 2_{(84}\right)$ & $99,{ }_{4}{ }_{(139)}$ \\
\hline
\end{tabular}

(1) $: 0,01<\mathbf{P}<0,05$. 


\section{4. - Conditionnement de la semence}

Chez les brebis et chez les agnelles, la fertilité après insémination pendant l'œstrus induit ( 2 IA/ 9,48 et 60 heures après arrêt du traitement) est plus élevée lorsque le sperme est conditionné en petit volume $(73,5 \mathrm{p}$. Ioo de MB avec 0,2 $\mathrm{ml}$ vs 64,7 p. Ioo avec $0,4 \mathrm{ml}$ ). Cette différence n'est cependant pas significative (tabl. 4).

\section{5. - Age des femelles}

Les trois paramètres définis plus haut varient beaucoup avec l'âge des femelles (fig. I). Le tracé des courbes qui les représentent évolue d'ailleurs dans le même sens. C'est chez les sujets les plus jeunes ( $\mathrm{I}, 5$ à 3,5 ans) que les valeurs sont les plus élevées, le maximum étant atteint à l'âge de 3,5 ans :

— fertilité : 60,7 p. Ioo pour les brebis de I,5 à 3,5 ans vs 52,0 p. Ioo pour cell:s de plus de 3,5 ans, $P<0,01$;

- prolificité : I58,5 p. IOO vs I5I,9 p. IOO, $\mathrm{P}>0,05$;

— fécondité : 96,2 p. Ioo vs 79,0 p. Ioo.

\section{III. - DISCUSSION}

Au cours de la saison sexuelle, la fertilité et la fécondité des brebis inséminées artificiellement sont plus faibles pendant un cestrus induit par un progestatif que pendant un cestrus naturel (tabl. I).

Cette subfertilité ne peut résulter d'une apparition incomplète des chaleurs. On sait en effet, qu'à l'époque où les animaux sont en pleine activité sexuelle, le pourcentage de venues en chaleurs après traitement hormonal est très élevé (THIMONIER et al., I $968 b$; CoGNIE et al., I970). Elle ne provient pas non plus de la semence, puisqu'en $\mathrm{O}_{1}$ et $\mathrm{O}_{2}$ les géniteurs sont les mêmes et les quantités de spermatozoïdes par animal identiques. Par ailleurs, la fécondance du sperme est satisfaisante comme en témoignent les résultats enregistrés en $\mathrm{O}_{2}$ (7I,8 p. Ioo de MB). Ceci montre qu'en dépit de bonnes conditions expérimentales, l'application du progestatif seul se traduit toujours par une fertilité plus faible pendant la chaleur artificielle.

Après injection de PMSG, par contre, le taux de fécondation est sensiblement augmenté et l'on parvient à un niveau de fertilité et de fécondité comparable à celui d'un oestrus naturel. L'hormone sérique apparaît donc comme le complément indispensable à tout traitement à l'acétate de fluorogestone, même pendant la saison sexuelle. Féconder dans un intervalle de $\mathrm{r} 2$ heures, au moins autant de brebis qu'on en féconderait en un cycle de I7 jours dans les conditions naturelles, et recueillir un nombre d'agneaux supérieur, tel est l'intérêt essentiel de cette méthode. Donnons à titre indicatif, les résultats obtenus, au second œstrus avec saillie naturelle, dans les élevages où notre expérience (tabl. 2 et 3 ) s'est déroulée :

— fertilité : 55,0 p. Ioo (des 283 brebis déjà inséminées et non fécondées)

- prolificité : 136,8 p. roo;

— fécondité : 76,0 p. Ioo (contre II 8 ,o dans notre meilleur traitement (§ 3$)$ ). 
TABI,EAU 4

Effet du conditionnement (paillette moyenne ou fine) de la semence sur la fertilité après traitement progestatif et $P M S G$

$\left(250 \times\right.$ ro $^{6} \mathrm{spz} /$ dose dans tous les cas)

\begin{tabular}{c|c|c}
\hline \multirow{2}{*}{ Animaux } & \multicolumn{2}{|c}{ Volume de sperme déposé } \\
\cline { 2 - 3 } & $0,4 \mathrm{ml}$ & $0,2 \mathrm{ml}$ \\
\hline Agnelles & $\left.74^{\prime}, 4_{(19}\right)$ & $87,5_{(18)}$ \\
\hline Brebis & $61^{\prime} 5_{(65)}$ & $70,5_{(78)}$ \\
\hline Total & $66^{\prime}, 7_{(82)}$ & $73,5(94)$ \\
\hline
\end{tabular}

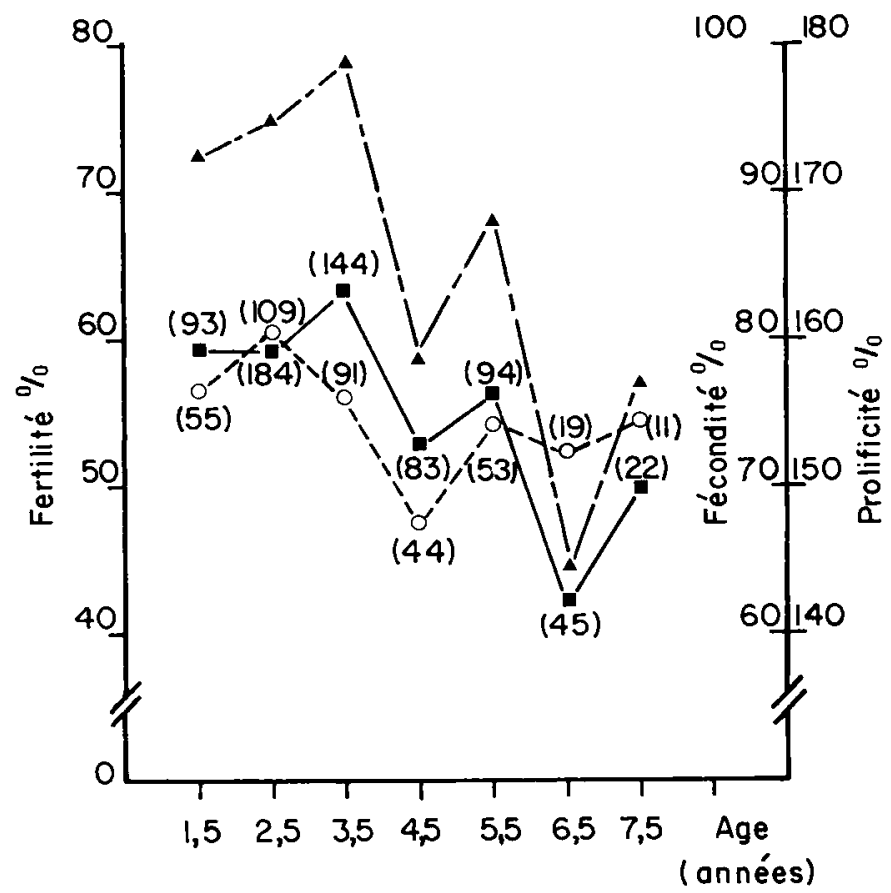

FIG. I. - Relation entre l'âge et la fertilité, la prolificité et la fécondité chez la brebis laitière Lacaune inséminée artificiellement après synchronisation des chaleurs pendant la saison sexuelle (les brebis provenant de Io élevages reçoivent 400 UI de PMSG)

( ) : animaux inséminés ou mettant bas

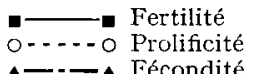


L'action synergique de la PMSG semble avoir trois causes :

- une synchronisation plus précise des chaleurs et de l'ovulation (NEwTON, I967; Cognie et al., I968, I970),

- une augmentation de la durée des chaleurs (BoLAND, I97I cité par Gordon, I972), dont l'effet retentit sur la fertilité (CoLAs, I968),

- une élévation du taux d'ovulation (Gordon, I967, NEwTon et BETTs, I966) dont dépend également, au moins à l'intérieur de certaines limites, la fertilité chez les ovins (HULET et Foote, I967).

L'augmentation simultanée de la fertilité et de la prolificité due à l'injection de PMSG traduit la relation qui existe chez les ovins entre ces deux critères. On la retrouve d'ailleurs à propos de la dose de FGA (tabl. 2), du moment d'insémination (tab1. 3) et de l'âge des brebis (fig. I et commentaires).

La dose de progestogène a une grande incidence sur les paramètres de reproduction. Elle constitue le second élément important de réussite de la méthode. Nos observations ne confirment pas celles de RoBInson (I970) qui n'enregistre aucun effet de la dose de FGA sur la fertilité. En fait, les conditions dans lesquelles cet auteur a opéré diffèrent des nôtres : pas de PMSG, une seule insémination déterminée d'après l'apparition des chaleurs, brebis non traites. Par contre, ce même auteur observe des venues en chaleur différentes selon les quantités de FGA utilisées. Bien que nous n'ayons pu procéder à un tel contrôle, il semble bien, au vu des résultats, qu'il en soit de même ici : pour obtenir des taux de fertilité très voisins $(60, \mathrm{I}$ p. Ioo et $63,8 \mathrm{p}$. Ioo) il est nécessaire d'inséminer deux fois avec $40 \mathrm{mg}, 3$ fois avec $30 \mathrm{mg}$. Ceci peut être interprété comme la conséquence d'une apparition des œstrus plus étalée avec les doses faibles (30 $\mathrm{mg}$ ).

Le moment d'insémination influence non seulement la fertilité mais aussi la prolificité et la fécondité du troupeau : c'est en inséminant tous les animaux 2 jours et 2,5 jours après le retrait de l'éponge qu'on en féconde le plus grand nombre ( 70 p. roo environ) et que la taille de la portée est maximum. C'est dire l'importance que revêt le choix du moment d'insémination dans la réussite d'une méthode qui ne comprend aucune détection de chaleurs.

Les taux de mises-bas des lots $36-48 \mathrm{~h}$ et $36-60 \mathrm{~h}$ sont à peu près identiques. On a donc autant de chances de féconder une brebis 48 heures que 60 heures après le retrait de l'éponge. Si l'on tient compte, d'une part de l'apparition et de la durée des chaleurs dans le cas d'un traitement FGA et PMSG, d'autre part de l'intervalle de temps séparant l'ovulation des premiers signes d'œestrus ( 29 heures selon CoGNIE et al., I970), il est logique de penser qu'une seule intervention (avec un nombre suffisant de spermatozoïdes) effectuée à 54 heures ne doit pas diminuer sensiblement la fertilité tout au moins pendant la saison sexuelle. Les travaux de Robinson et al. (I970) permettent aussi de le penser.

Le conditionnement de la semence en petites fractions $(0,2 \mathrm{ml})$ augmente la fertilité. Ce résultat est en accord avec ceux d'AlIISON et RoBInson (I97I). Par ailleurs, l'utilisation de la paillette de petit diamètre pour de telles fractions présent: l'avantage d'une mise en place plus précise et plus profonde du sperme dans le col utérin. LiGHTFOOT et RESTALI, (I97I) ont montré qu'on peut ainsi augmenter le taux de fécondation.

Dans les conditions naturelles, c'est-à-dire lorsque les animaux ne reçoivent 
aucune préparation hormonale, les sujets âgés de un à deux ans, sont généralement les moins productifs (faible taux de venue en œstrus, fertilité plus faible, prolificité plus basse). Ce phénomène se rencontre aussi bien chez les races à viande (PRuD'HON et al., I968) que chez les races laitières (CoLAS, I967 non publié). Après traitement, au contraire, leur capacité de reproduction augmente beaucoup pour atteindre, voire même dépasser, celle des animaux âgés. Cela présente un intérêt pratique car dans les élevages leur effectif est généralement supérieur à celui des femelles plus vieilles (environ 65 p. roo) (1).

Sur le plan physiologique, ces résultats peuvent être interprétés comme la conséquence d'un seuil de sensibilité aux hormones gonadotropes, variable selon les âges. A dose égale, les réponses sont plus marquées chez les jeunes sujets.

Ces observations, que 1'on peut rapprocher de celles d'HuLET et STORMSHaK (I972), laissent supposer que l'on pourrait améliorer l'efficacité de ces méthodes en ajustant la dose de PMSG à l'âge des animaux.

\section{CONCLUSION}

Nous avons constaté que :

- la PMSG administrée à la fin du traitement progestatif de synchronisation augmente la fertilité, la prolificité et la fécondité des brebis. Il existe une relation assez étroite entre la fertilité et la prolificité.

- les résultats dépendent de la dose de FGA, (la dose optimum étant de $40 \mathrm{mg}$ pendant la saison sexuelle), du moment d'insémination (48 et 60 heures après le retrait de l'éponge) et du conditionnement de la semence (paillette de petit diamètre contenant $0,2 \mathrm{ml}$ soit $250 \times 10^{6} \mathrm{spz}$ ).

- les performances de reproduction des femelles sont liées étroitement à l'âge. Contrairement à ce que l'on observe dans les conditions naturelles, "ce sont les sujets les plus jeunes $(I, 5$ à 3,5 ans) qui sont les plus productifs.

Reçu pour publication en juin 1973.

\section{REMERCIEMENTS}

Cette étude a pu être réalisée grâce à la collaboration des organismes professionnels (Confédération des Éleveurs de brebis laitières et des Industriels de Roquefort, Institut technique ovin et caprin) et des éleveurs. Nous tenons à les en remercier. Notre reconnaissance s'adresse également aux personnes du C. R. V. Z. de Nouzilly et de l'École nationale supérieure agronomique de Montpellier, qui ont bien voulu prêté leur concours et à Mademoiselle Solari du C. N. R. Z. de Jouy en Josas pour les conseils qu'elle nous a donnés dans l'interprétation statistique des résultats.

(1) Ce chiffre nous a été communiqué par la Confédération des Éleveurs de brebis et des Industrieis de Roquefort (12 - Millau). 


\section{SUMMARY}

\section{FERTILITY, PROLIFICACY AND FECUNDITY DURING \\ THE BREEDING SEASON OF EWES ARTIFICIALIY INSEMINATED AFTER TREATMENT WITH FLUOROGESTONE ACETATE,}

With the aim of overcoming the reported decrease in fertility after synchronisation of oestrus and artificial insemination in sheep a series of experiments was designed in which the efficacy of the hormone treatment and the conditions of artificial insemination were examined.

A total of I 436 dry females of the meat breeds "Ile-de-France " and "Prealpes" and the milk breed "Lacaune " were studied during the breeding scason and the following treatments were applied :

I. Intravaginal sponges with either 30 or $40 \mathrm{mg}$ fluorogestin acetate (FGA).

2. An injection of PMSG at the time of withdrawal of the sponges, or no PMSG (where PMSG was injected, adult ewes reccived 4 OO IU and maiden ewes 500 IU).

3. Insemination at the first synchronised ostrus one cycle later.

4. The age of the ewe.

5. Insemination following detection of ostrus by teaser rams or insemination on a time basis after the withdrawal of the sponges, comencing at either 36 or $4^{8}$ hrs. (In the case of the second ostrus ewes were inseminated once per day on the $I 7^{\text {th, }} 18$ th, Igth and 20 th days).

6. Two inseminations per ewe or three. In every case the dose was $25^{\circ} \times 1^{6}$ sperm cells per insemination. The interval between inseminations was either 12 or 24 hours.

7. An inseminate of either $0.40 \mathrm{ml}$ in wide diameter straws or $0.20 \mathrm{ml}$ in narrow diameter straws (which gave better penetration of the cervix). The sperm was diluted with reconstituted skim milk and stored in straws at $+15^{\circ} \mathrm{C}$ for about $8 \mathrm{hrs}$.

Ewes treated with PMSG had a higher level of fertility and fecundity than those not receiving PMSG but the prolificacy was unaltered. When PMSG was given, the fertility and fecundity were identical at both the Irst and second cycle.

Fertility, fecundity and prolificacy were higher with sponges containing $4^{\circ} \mathrm{mg}$ FGA than those containing $30 \mathrm{mg}$ FGA $(6 \mathbf{1} .7,161.0,99.0$ vs $53.3,149.5,79.7$ respectively).

The most favourable combination was 2 inseminations at $4^{8}$ and $60 \mathrm{hr}$ after the withdrawal of the sponges. A third insemination did not augment the reproductive performance.

A volume of $0.2 \mathrm{ml}$ semen administered in fine inscminating straws was more effective than $0.4 \mathrm{ml}$ in wider straws $(73.5$ vs $64.7 ; \mathrm{P}>0.05)$. In the case of the Lacaune ewes all aspects of reproductive performance of young ewes (I.5-3.5 years) were better than those of older ewes (> 3.5 years) $(60.7,158.5,96.2$ vs $52.0,151.9,79.0$ respectively).

\section{RÉFÉRENCES BIBLIOGRAPHIQUES}

Alisson A. J., Robinson T. J., I97I. Fertility of progestagen treated ewes in relation to the number and concentration of spermatozoa in the inseminate. Austr. J. Biol. Sci., 24, roor-Ioo8.

Cognie Y., Thimonier J., Mauleon P., r968. Étude du moment d'ovulation chez la brebis après traitement progestatif administré par voie vaginale et injection de PMSG, pendant la période d'anoestrus saisonnier. Ve Congr. Intern. Reprod. anim. insem. artif., Paris, vol. II, I403-I406.

Cognie Y., Mariana J. C., Thimonier J., I97o. Étude du moment d'ovulation chez la brebis normale et traitće par un progestagène associé ou non à une injection de PMSG. Ann. Biol. anim. Bioch. Biophys., 10, $15-24$.

Colas G., Dauzier L., Courot, M., Ortavant R., Signoret J. P., rg68. Résultats obtenus au cours de I'étude de quelques facteurs importants de l'insémination artificielle ovine. Ann. Zootech., 17, $47-57$.

Colas G., I968. Durée de l'œestrus et fertilité après insémination artificielle chez la Brebis. VI Congr. Intern. Reprod. Anim. Insem. artif., Paris, vol. II, rolo-rorg.

Desvignes A., Darpoux R., I964. Valeurs d'élevages des brebis utilisées pour le traitement industriel avec différentes races de béliers. Bull. Techn. Inf., 195, 293-90I. 
GoRDon I., I967. Induction of early breeding in sheep by standard and modified progestagen PMS treatments. J. Agric. Sci. Camb., r6, 337-341.

Gordon I., 1972. Control of reproduction in sheep: towards programmed lamb production. Departement's $J$. Vol. LXVIII.

Hawk H. W., Conley H. H., r97r. Loss of spermatozoa from the reproduction tract fo the ewe and intensification of sperm "breakage " by progestagen. J. Reprod. Fert., 27, 337-347.

Hulet C. V., STORMShak F., I972. Some factors affecting response of anoestrous ewes to hormone treatment. J. Anim. Sci, 34, Ior r-rory.

Hulet C. V., Foote W. C., 1967. Relationship between ovulation rate and reproduction performance in sheep. J. Anim. Sci, 26, 563-566.

Lightfoot R. J., Restall B. J., I97I. Effects of site of insemination, sperm motility and genital contraction on transfert of spermatozoa in the ewe. J. Reprod. Fert., 26, I-3.

Lindsay D. R., Moore N. W., Robinson T. J., Salamon S., Shelton J. N., I967. The evaluation of an oral progestagen (Provera, MAP) for the synchronisation of oestrus in the entire cyclic Merino ewe Paper I in : "The control of the ovarian cycle in the sheep ", Ed. T. J. Robinson, Sydney N. S. W., Sydney University Press, 3-17.

Lison L., I958. Statistique appliquée à la biologie expérimentale. La planification de l'expérience et l'analyse des résultats. Gauthiers-Villars Edit., $346 \mathrm{p}$.

Newton J. E., 1967. Effects of hormonal synchronisation and PMS on breeding in ewes. Vet. Rec. $81,422-425$.

Newton J. E., Betrs J. E., I966. Factors affecting litter-size in the Scotch Half-herd ewe. I. Treatment with PMS and progesterone. J. Reprod. Fert., 12, I67-I75.

Prud'hon M., Denoy I., Desvignes A., Goussopoulos J., ig68. Étude des résultats de six années d'élevage des brebis Mérinos d'A rles du domaine du Merle. II. Relation entre l'âge, le poids, l'époque de lutte des brebis et les divers paramètres de la fécondité. Ann. Zootech., 17, 31-45.

Quinlivan T. D., Robinson T. J., 1967. The number of spermatozoa in the fallopian tubes of ewes at intervals after artificial insemination following withdrawal of $\mathrm{SC} 9880$ impregnated intravaginal sponges. Paper XIII, in : The control of the ovarian cycle in the sheep, Ed. T. J. Robinson, Sydney N. S. W., Sydney University Press, I77-194.

Quinlivan T. D., Robinson T. J., Ig69. Numbers of spermatozoa in the genital tract after artificial insemination of progestagen treated ewes. J. Reprod. Fert., 19, 73-86.

Robinson 'T. J., I965. Use of progestagen-impregnated sponges inserted intravaginally or subcutaneously for the control of cestrous cycle in the sheep. Nature (Lond.), 206, 39-40.

Robinson T. J., Lamond D., 1966. Control of reproduction in sheep and cattle. Proc. Austr. Soc. Anim. Prod., 6, IO-I8.

Robinson T. J., Moore N. W., Lindsay O. R., Fletcher I. C., Salamon S., igjo. Fertility following synchronization of cestrus in the sheep with intravaginal sponges. I. Effects of vaginal douche, supplementary steroids, time of insemination and numbers and dilution of spermatozoa. Austr. J. Agric. Res., 21, 767-78I.

Robinson T. J., I970. Fertility following synchronisation of oestrus in the sheep with intravaginal sponges. I. Effects of dose of progestagen and rate of absorption. Austr. J. A gric. Res., 21, 783-792.

Thimonier J., Mauleon P., Cognie Y., Ortavant R., I 968 a. Déclenchement de l'œstrus et obtention précoce de gestation chez les agnelles à l'aide d'éponges vaginales imprégnées d'acétate de fluorogestone. Ann. Zootech., 17, 275-288.

Thimonier J., Cognie Y., Colas G., Mauleon P., ig68 b. Synchronisation de l'ostrus chez les brebis à l'aide d'éponges vaginales imprégnées de progestagène ( I $~ \alpha$ acétoxy-9 $\alpha$ fluoro-I $\beta$ hydroxy-pregn-4-

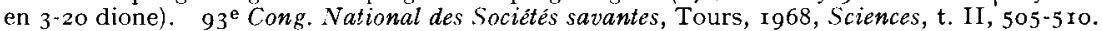

Willemse A. H., Brand A., Murling F., I968. Oestrus synchronisation in texel sheep. Titjdschr. Diergeneesk., 93, 66-82.

Wishart D. F., r967. Synchronisation of cestrus in sheep. The use of pessaries. Vet. Rec., 81, $276-287$. 